\title{
ACTUALIZACION DE LA BRONQUIOLITIS AGUDA
} ACUTE BRONCHIOLITIS UPDATE

\author{
Dr. Luis Enrique Vega-Briceño \\ Pediatra Broncopulmonar Clínica Alemana. Profesor Asociado de Pediatría UDD. Asesor Médico GSK.
}

\section{INTRODUCCIÓN}

Existen pocas condiciones respiratorias tan controvertidas como la bronquiolitis aguda (BA). No sólo ha despertado la pasión y confusión de muchos clínicos, sino que lo más consistente es la inconsistencia publicada en torno a ella. La BA es la infección del tracto respiratorio inferior más común en los niños menores de 2 años. En los Estados Unidos, es responsable de más de 100,000 hospitalizaciones/año en menores de 12 meses, siendo el virus respiratorio sincicial (VRS) el más frecuentemente identificado $(1,2)$. La BA tiene un importante impacto económico y sanitario, así un estudio reportó un costo promedio de $\$ 8.530$ paciente/ admisión, lo que representó $\$ 1.7$ billones a nivel país (1). Una proporción sustancial de niños experimentará al menos un episodio de BA y hasta el 2-3\% de todos los niños serán hospitalizados por BA antes de su primer cumpleaños (3).

A pesar de su elevada prevalencia, su diagnóstico carece de una definición internacional única. La Academia Americana de Pediatría (AAP) definió la BA como la presencia de "rinitis, taquipnea, sibilancias, tos, crepitaciones, uso de músculos accesorios y/ 0 aleteo nasal en niños", sin hacer alusión al concepto primer episodio (4). Resulta interesante las diversas nomenclaturas erróneas empleadas como sinónimos: Hiperreactividad bronquial bronquitis viral, neumonía atípica, debut de asma, entre otros (5). Independiente de ello, se reconoce que su diagnóstico es clínico, existiendo numerosos tratamientos ensayados con resultados muy diferentes debido a la inconsistencia en su definición (edad diagnóstica, presencia del VRS, puntajes clínicos, etc).

\section{ETIOLOGÍA}

Además del VRS hay otras causas virales: rinovirus (14-30\%), bocavirus humano (14$15 \%)$, metapneumovirus (3-12\%), enterovirus, adenovirus, coronavirus e influenza (1-8\%) (6), no existiendo diferencias clínicas relevantes entre ellos. La coinfección ocurre en el 20-30\% de los niños, pareciendo no estar asociada a una mayor gravedad $(7,8)$; sin embargo, algunos autores han sugerido que la coinfección de VRS con rinovirus o metapneumovirus se asocia a mayor morbilidad $(9,10)$. Existe una

RESUMEN

La bronquiolitis aguda es una condición respiratoria frecuente en los niños menores de 2 años. Representa la principal causa de hospitalización infantil y se caracteriza por la presencia de sibilancias asociada a signos de una infección respiratoria alta. El agente etiológico más común es el virus respiratorio sincicial. Existe una falta de consenso con respecto a su definición clínica; y por ello, su tratamiento varía en todo el mundo. El diagnóstico es clínico, sin necesidad de emplear laboratorio o imágenes en forma rutinaria. Diversas revisiones han demostrado que los broncodilatadores, adrenalina, corticoides y antibióticos, entre otros, carecen de eficacia por lo que no se sugiere su empleo. El tratamiento sigue siendo de soporte, mediante la administración de oxígeno y manteniendo una adecuada hidratación. Cuando no se logra disminuir el trabajo respiratorio o corregir la hipoxemia se puede utilizar la presión positiva en la vía aérea para prevenir y controlar la insuficiencia respiratoria. Este artículo desarrolla una breve revisión de las principales características clínicas, epidemiológicas, radiológicas, así como algunos de los diferentes tratamientos publicados en las últimas dos décadas.

Palabras claves: Bronquiolitis aguda, definición, diagnóstico, evaluación, tratamiento.

\section{ABSTRACT}

Acute bronchiolitis is a common respiratory condition in children under 2 years old. It represents the main cause of childhood hospitalization characterized by the presence of wheezing associated with signs of an upper respiratory infection. The most common etiologic agent is respiratory syncytial virus. There is a lack of consensus regarding its clinical definition; and therefore, its treatment varies around the world. Diagnosis is clinical, without the need for routine laboratory or imaging. Various reviews have shown that bronchodilators, epinephrine, corticosteroids, and antibiotics, among others, lack efficacy, so their use is not suggested. Treatment continues to be supportive, by administering oxygen and maintaining adequate hydration. When it is not possible to reduce the work of breathing or correct the hypoxemia, positive airway pressure can be used to prevent and control respiratory failure. This article develops a brief review of the main clinical, epidemiological, and radiological characteristics, as well as some of the different treatments published in the last two decades.

Keywords: Acute bronchiolitis, definition, diagnosis, assessment, treatment.

marcada estacionalidad, siendo más prevalente durante los meses de invierno (3,4). La infección por el VRS no otorga inmunidad permanente, siendo frecuente la reinfección a lo largo de toda la vida (3). Con el uso de las pruebas de amplificación del ácido nucleico ha mejorado la capacidad para detectar diferentes virus; detectándose en el $30 \%$ de los menores de 6 años asintomáticos, lo cual puede ser debido a una colonización asintomática, incubación antes de la infección clínica o la diseminación prolongada después de una infección (11,12).

\section{FISIOPATOLOGÍA}

La BA se caracteriza por la inflamación extensa y aumento en la producción de moco asociado a necrosis de las células epiteliales de los bronquiolos (13). El VRS infecta las células epiteliales nasofaríngeas donde se replica y luego de 2-3 días se disemina a la vía aérea inferior. Esta inflamación se caracteriza por una infiltración mononuclear peribronquial y edema de la submucosa, pudiendo el daño tisular ocurrir por una lesión viral directa o indirectamente por la activación de la respuesta inmune (6). El daño y la necrosis tisular pueden causar obstrucción parcial o total del flujo de aire con atrapamiento de aire distal, atelectasias y alteración de la relación ventilación-perfusión, responsable final de la hipoxemia y aumento del trabajo respiratorio $(4,6)$. El broncoespamo parece desempeñar un papel menor en la fisiopatología de la BA a diferencia del asma $(3,6)$.

\section{CARACTERÍSTICAS CLÍNICAS}

A menudo la BA se inicia con signos de una infección respiratoria alta (rinorrea clara,

Autor para correspondencia: Dr. Luis Enrique Vega-Briceño Email: levega@udd.cl 
fiebre baja) la cual progresa gradualmente con taquipnea, sibilancias y tos. En los menores de 3 meses y niños con antecedente de prematurez la apnea puede ser el único síntoma, siendo común el rechazo alimentario (6). En los niños más pequeños es frecuente auscultar crépitos finos inspiratorios mientras que las sibilancias espiratorias son más frecuentes en los niños mayores (3). En general existe aumento del trabajo respiratorio (polipnea, respiración abdominal, uso de músculos accesorios), puede presentarse con compromiso del estado general y cianosis. No existe un puntaje clínico único validado para evaluar la gravedad (14)

En pacientes ambulatorios la resolución de los síntomas se observa más allá de los 14 días en el $40 \%$ de los niños y sólo el $10 \%$ continúa con síntomas después de 4 semanas (5). La mediana de días de síntomas previos a la hospitalización es de 4 (rango 2-5) días, sin

Tabla 1. Factores de riesgo para desarrollar bronquitis aguda.

\begin{tabular}{|l|}
\hline GÉNERO MASCULINO \\
\hline PREMATUREZ \\
\hline MENOR DE 3 MESES \\
\hline NACEREN INVIERNO \\
\hline DISPLASIA BRONCOPULMONAR \\
\hline ENFERMEDAD NEUROMUSCULAR \\
\hline CARDIOPATÍA CONGÉNITA \\
\hline EXPOSICIÓNA TABACO \\
\hline ELEVADA PARIDAD \\
\hline CORTA DURACIÓN DELALACTANCIA \\
\hline MADRE ADOLESCENTE \\
\hline ASMA MATERNA \\
\hline BAJO NIVEL SOCIOECONÓMICO \\
\hline
\end{tabular}

Tabla 2. Diagnósticos diferenciales de la bronquiolitis aguda.

\begin{tabular}{|c|}
\hline REFLUJO GASTROESOFÁGICO \\
\hline TRAQUEO O BRONCOMALACIA \\
\hline ASPIRACIÓN DE CUERPO EXTRAÑO \\
\hline ANILLO VASCULAR \\
\hline MASAS O TUMORES MEDIASTÍNICOS \\
\hline $\begin{array}{l}\text { ENFERMEDADES CARDIOPULMONARES } \\
\text { CONGÉNITAS }\end{array}$ \\
\hline MIOCARDIOPATÍA \\
\hline
\end{tabular}

existir asociación con la duración de la hospitalización ni ventilación con presión positiva (15). La estadía hospitalaria promedio es de 1 día (rango: 0-3), aunque otros reportaron un promedio de 3,3 días (desviación estándar: 2,8) (16). Los factores de riesgo para presentar BA se detallan en la tabla 1 (15). Si bien existen distintos fenotipos clínicos con una amplia heterogeneidad, algunos autores sugieren que ésta sería la responsable de las diferentes respuestas a los tratamientos médicos ensayados (17).

\section{DIAGNÓSTICO DIFERENCIAL}

En la mayoría de los casos, el diagnóstico de BA es evidente. Sin embargo, existen otras condiciones respiratorias con presentaciones atípicas que presentan dificultad respiratoria y síntomas recurrentes, pero sin signos de una infección respiratoria viral aguda (Tabla 2) $(3,4)$. La sospecha de asma debe considerarse en los lactantes mayores con episodios recurrentes de sibilancias.

\section{EVALUACIÓN CLÍNICA}

El diagnóstico de BA es clínico y deben identificarse los factores de riesgo asociados con un curso más severo como son la edad temprana, que se asocia con un mayor riesgo de apnea, hospitalización prolongada, hipoxemia, ingreso en una UCl y necesidad de ventilación mecánica (4). La oximetría de pulso debe incluirse en toda evaluación clínica de BA, ya que permite detectar la hipoxemia que no detecta el examen clínico. El curso de la BA es variable y deben realizarse evaluaciones repetidas, especialmente en lactantes con factores de riesgo $(3,4)$. En los pacientes ambulatorios, la disminución leve de la Sp02 (93-95\% con aire ambiente) no predice la progresión de la enfermedad o la reconsulta al servicio de urgencia (18)

\section{LABORATORIO}

No se ha demostrado que las pruebas de laboratorio tengan un impacto sustancial en el curso clínico de la BA (19). La aplicación de guías clínicas ha reducido el uso de laboratorio, tratamientos y costos asociados (20); por otra parte, la identificación viral demostró reducir la estadía hospitalaria (21) y las infecciones intra-hospitalarias (10). El hemograma y la PCR carecen de valor clínico en la mayoría de los pacientes, aunque deben ser solicitados cuando se sospeche una sobreinfección bacteriana $(3,4)$. Los electrolitos en sangre deben solicitarse frente a una mala alimentación y signos de deshidratación. Los gases arteriales están justificados frente a una dificultad respiratoria grave y/o insuficiencia respiratoria $(3,4)$.

\section{RADIOGRAFÍA DE TÓRAX (RX)}

Muchos lactantes con BA tienen alteraciones radiológicas que carecen de significado clínico y que no se correlacionan con la gravedad de la enfermedad (4); por ello, la Rx puede ser considerada en el paciente hospitalizado con fiebre prolongada, ingreso a UCl, ventilación mecánica, Sp02 <90\%, enfermedad cardiopulmonar crónica o frente a la sospecha de otro diagnóstico (Figura 1) (22,23). La presencia de condensación y atelectasia se asocia con mayor riesgo de gravedad (22). En estudios prospectivos, los niños a los que se solicitó una Rx tuvieron más probabilidad de recibir antibióticos $(23,24)$.

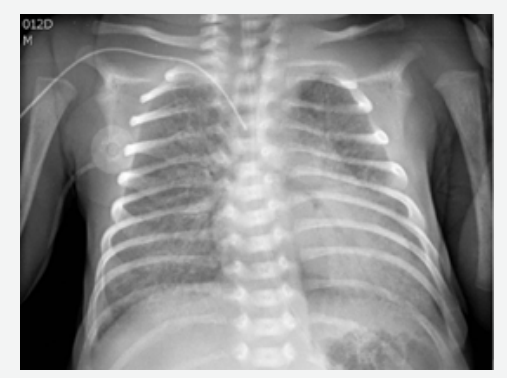

Figura 1. Radiografía de tórax de lactante con diagnóstico de bronquiolitis aguda grave. Se observa gran hiperinsuflación, compromiso intersticial difuso bilateral y condensación con disminución de volumen y broncograma aéreo de LII (retrocardio)

\section{TRATAMIENTO}

Diversos estudios han mostrado una amplia variación en el tratamiento. En Estados Unidos (25), Canadá (26) y Europa (27) se observaron variaciones que se correlacionaron más con las preferencias individuales o institucionales que con la gravedad del paciente.

\section{Oxígeno (02)}

No existe consenso sobre el nivel de Sp02 a alcanzar con el soporte de 02 administrado mediante cánula nasal o mascarilla $(28,29)$. En el Reino Unido, se suele administrar 02 para alcanzar una Sp02 del 92-95\%, mientras que la AAP recomienda mantener la Sp02 en al menos $90 \%$ (28). Sin embargo, estudios observacionales sugieren que una $\mathrm{SpO} 2$ de $90 \%$ versus $94 \%$ reduce significativamente la duración de la estadía hospitalaria, por ello las guías de la AAP recomiendan un nivel reducido de Sp02 a medida que los lactantes mejoran. 


\section{Hidratación y alimentación}

El aumento del trabajo respiratorio puede causar una alimentación inadecuada y conducir a la deshidratación. La polipnea y fiebre aumentan la pérdida de líquidos; así cuando esta es mayor a 60-70 respiraciones por minuto la alimentación puede verse comprometida, especialmente si existen abundantes secreciones nasales, aumentando el riesgo de aspiración pulmonar (31). En pacientes ambulatorios la alimentación puede mantenerse en forma fraccionada y promoviendo la lactancia materna. En cambio, en los hospitalizados se puede necesitar la suplementación de líquidos por vía intravenosa (IV) o mediante sonda gástrica (SNG) (32). La ventaja del IV es el menor riesgo de aspiración y la no interferencia con las nariceras de 02 (33), pero su desventaja es la baja entrega de calorías, el mayor riesgo de sobrecarga de líquidos y desequilibrio electrolítico (33). La alimentación por SNG permite asegurar un mejor estado nutricional y equilibrio de nitrógeno, incluso permitiendo el uso de leche materna. Si bien no existe evidencia a favor de IV vs SNG en niños con BA (34), muchos promueven la alimentación por SNG (35). Se recomiendan cubrir la pérdida y evitar la deshidratación sin exceder el $100 \%$ de las necesidades diarias $(100 \mathrm{ml} / \mathrm{kg} /$ día para los menores de $10 \mathrm{~kg}$ ). Esta descrita la retención de líquidos debido a la secreción inapropiada de hormona antidiurética; por lo que se puede emplear el $70-80 \%$ de las necesidades hídricas diarias, especialmente en los más graves. En ellos se requiere la monitorización estrecha del peso, osmolalidad sérica/urinaria y los electrolitos séricos (36).

\section{Salbutamol}

Los estudios de pacientes hospitalizados no han mostrado cambio en la evolución clínica que justifique el uso de salbutamol. Si bien existen grandes diferencias metodológicas que dificultan la comparación, la revisión Cochrane identificó 30 estudios que evaluaron el uso de salbutamol en BA; 21 de ellos no encontraron beneficio en los niños hospitalizados (37). En los pacientes ambulatorios, la Sp02, la tasa de admisión al hospital o el tiempo hasta la resolución de los síntomas fue igual al grupo placebo.

\section{Adrenalina \\ El empleo de adrenalina está muy difun- dido entre los pediatras. Una revisión no en- contró ningún beneficio versus el placebo en acortar la duración de la estadía hospitalaria, pudiendo incluso prolongarla (38). En los pa- cientes ambulatorios, se encontró una dismi- nución del número de hospitalizaciones cuan-}

do se administró en el servicio de urgencia, sin alterar el curso de la enfermedad. Si bien la evidencia no justifica el uso rutinario, la experiencia clínica sugiere que en algunos lactantes existe una mejoría clínica después de su administración, por lo que parece razonable probarlo y evaluar la respuesta en forma individual y documentar los cambios de manera objetiva. No se debe administrar adrenalina a niños con BA hospitalizados.

\section{Bromuro de Ipatropio}

No se ha demostrado que los anticolinérgicos alteren el curso de la BA. Aunque un pequeño grupo de pacientes individuales pueden mostrar una respuesta clínica favorable, los estudios clínicos controlados han demostrado que no existe diferencia versus el placebo. No se recomienda el uso de bromuro de ipatropio en lactantes con BA (38).

\section{Corticoides}

Hasta el $60 \%$ de los niños por BA reciben corticoides sistémicos (25) sin existir evidencia que respalde su uso. Inicialmente se demostró una disminución en la duración de la estadía hospitalaria de -0,38 días, sin ser diferente al grupo control (25). Posteriormente, otra revisión (39) que incluyó solo estudios con niños menores de 2 años y su primer episodio de sibilancias asociados a síntomas de una enfermedad viral, no mostró reducción en la tasa de hospitalización, puntajes clínicos, permanencia en el servicio de urgencia ni en el tiempo de resolución de los síntomas; sin reducir tampoco la duración de la estadía hospitalaria. Un estudio demostró beneficio al usar dexametasona en altas dosis $(0,6 \mathrm{mg} / \mathrm{kg} /$ día durante $48 \mathrm{~h})$ en niños con ventilación mecánica (40), así como con la terapia combinada de adrenalina inhalada y dexametasona oral en dosis altas $(0,6$ $\mathrm{mg} / \mathrm{kg} /$ día por 5 días), reduciendo la tasa de ingresos hospitalarios (41). Muchos médicos con frecuencia consideran los antecedentes personales o familiares de asma y alergias para decidir el inicio de un corticoide en BA sin existir evidencia que muestre algún beneficio (42). En cuanto al uso de corticoides inhalados no existe beneficio en el curso de la enfermedad (43).

\section{Solución Salina Hipertónica (SSH)}

Se reconoce que la SSH reduce el edema de los bronquiolos y el taponamiento mucoso, mejora el aclaramiento mucociliar y rehidrata el líquido de la superficie de las vías respiratorias en pacientes con fibrosis quística. Muchas de estas propiedades teóricas han sido extrapoladas a pacientes con BA. Los estudios iniciales mostraron una reducción en la estadía hospitalaria, mejorando transitoriamente los puntajes clínicos (44), aunque posteriormente aparecieron resultados contradictorios $(45,46)$. Dos revisiones con 24 y 15 ensayos $(47,48)$ demostraron una diferencia significativa en la duración de la hospitalización a favor del grupo que recibió SSH (-0.45 días y -0.36 días, respectivamente). En siete estudios, la SSH redujo el riesgo de hospitalización en $20 \%$, sin reportar efectos adversos significativos. Otra revisión que eliminó los estudios ambulatorios y consideró la dispersión de los días de síntomas (49) concluyó que la SSH no reduce la duración de la estadía hospitalaria (-0.21 días; IC del 95\%:-0.43 a -0 .02). En un análisis de 3200 pacientes provenientes de 24 ensayos clínicos se encontró que el riesgo de hospitalización disminuyó en $20 \%$ vs el grupo control; por ello, los autores concluyeron que el uso de SSH es seguro y potencialmente útil en niños con BA (50)

\section{Sulfato de magnesio}

Una revisión que incluyó 4 estudios controlados concluyó que no existe suficiente evidencia para establecer la eficacia y seguridad del sulfato de magnesio para el tratamiento de niños con BA, sin existir diferencias en el tiempo de recuperación, duración de ventilación mecánica y estadía en la UCI (51). Se requieren estudios controlados para evaluar el tiempo de recuperación, alta hospitalaria y eventos adversos.

\section{Ribavirina}

Las opiniones en torno al uso de ribavirina para la BA son controvertidas. Una revisión con 11 ensayos clínicos controlados (52), evaluó el efecto de la ribavirina en la fase aguda: sólo 4 demostraron mejorar la Sp02 y acortar la estadía hospitalaria, mientras que otros 3 sólo demostraron mejoría de los puntajes respiratorios. Los diseños empleados en los distintos estudios son muy variados e inconsistentes. La terapia con ribavirina ofrece un beneficio muy marginal (si es que existe) la mayoría de las veces. Los requisitos asociados al traslado y manipulación, los riesgos potenciales en la salud del personal y su elevado costo desalientan su empleo (53); sin embargo, puede ser considerado en situaciones muy seleccionadas que involucran BA grave por VSR o en aquellos con riesgo de desarrollar una enfermedad grave (Ej. Inmunosupresión).

\section{Antibióticos}

Con frecuencia existe el temor a la fiebre elevada, la corta edad de un niño o el riesgo de una infección bacteriana asociada. Muchos estudios han demostrado una baja tasa de infección bacteriana grave (IBG) $(0-3,7 \%)$ 
en pacientes con BA o VRS positivo; y cuando ocurría, la infección urinaria (ITU) era la más frecuente $(70 \%)$ en lugar de una bacteriemia 0 meningitis (54-56). Un estudio en niños febriles menores de 2 meses (54) demostró mayor riesgo de IBG en neonatos, independiente del resultado del VRS. Hasta el $25 \%$ de los lactantes hospitalizados por BA tienen atelectasia o infiltrados confluyentes a menudo interpretados como neumonía bacteriana (57). Si bien la otitis media aguda (OMA) en los niños puede ser causada por el VRS, no existen características clínicas que permitan diferenciarla de una infección bacteriana. Un estudio (58) identificó OMA en el $53-62 \%$ de los lactantes con $B A$, siendo los patógenos más frecuentes el $S$. pneumoniae, $H$. influenzae y $M$. catarrhalis aislados mediante punción timpánica, sin que ella influya en el curso clínico ni en los resultados del laboratorio. Los macrólidos tienen propiedades antiinflamatorias conocidas. Dos ensayos controlados no encontraron diferencias entre azitromicina y placebo en la duración de la estadía hospitalaria, la necesidad de oxígeno o el reingreso hospitalario $(59,60)$. Un estudio demostró reducir las concentraciones nasales de IL-8 prolongando el tiempo libre de síntomas (61). Dada la poca evidencia, no se recomienda el uso rutinario de macrólidos para el manejo de la BA.

\section{Fisioterapia}

La BA se asocia con edema de las vía aérea y desprendimiento del epitelio respiratorio dando lugar a un atrapamiento de aire e hiperinsuflación. Las atelectasias segmentarias son más características que las lobares. Una revisión Cochrane (62) encontró 3 ensayos controlados que evaluaron la fisioterapia torácica en pacientes hospitalizados con bronquiolitis, sin encontrar ningún beneficio clínico con el uso de técnicas de vibración y percusión (63). La succión de las fosas nasales puede proporcionar un alivio temporal de la congestión nasal. No hay evidencia que apoye la succión profunda de rutina de la faringe inferior o la laringe.

\section{Ventilación no invasiva}

La presión positiva continua en la vía aérea (CPAP) mediante una interfase nasal se ha utilizado en niños con BA moderada-grave. El CPAP permite el reclutamiento alveolar, lo que reduce la resistencia media de la vía aérea reduciendo la hiperinsuflación, el trabajo respiratorio y favoreciendo el intercambio de gases. El CPAP (4-8 cm H2O) reduce la PCO2 y dificultad respiratoria en forma rápida y segura, aunque no ha demostrado que reduzca la necesidad de ventilación invasiva $(64,65)$. El uso de cánulas nasales de alto flujo humidificadas
(HFNC) es una alternativa al uso del CPAP (66). Actúa incrementando la presión faríngea y reduciendo el trabajo respiratorio, disminuyendo la necesidad de intubación (67). La HFNC puede tolerarse mejor que el CPAP nasal (68). Una revisión del 2014 concluyó que no existían pruebas suficientes para determinar la eficacia de HFNC en BA (69). Si bien existe reporte del síndrome de fuga de aire en casos aislados (70), la seguridad del CPAP y HFNC permite su uso temprano en niños con BA moderada, siempre y cuando no se retrase el inicio de una ventilación mecánica convencional (71). La elección de la terapia a utilizar dependerá de la gravedad del paciente, la complejidad del centro asistencial y la experiencia en los sistemas de ventilación no (72).

\section{SEGUIMIENTO}

Los niños hospitalizados con BA tienen mayor riesgo de: a) desarrollar asma, b) pérdida de función pulmonar, c) incremento de la hiperreactividad bronquial (73). El mayor riesgo de asma se encuentra particularmente en los hospitalizados con BA por VRS negativa o BA por rinovirus, disminuyendo esta asociación con la edad; aunque un estudio demostró incremento del riesgo de atopia (74-76). La asociación entre BA y el desarrollo de asma es compleja y probablemente esté relacionada con mecanismos genéticos, estructurales, inmunológicos, inflamatorios y ambientales.

\section{CONCLUSIONES}

El término BA debería emplearse únicamente en los menores de 2 años. Su diagnóstico es clínico, por lo que no requiere exámenes de laboratorio o imágenes en forma rutinaria. Si bien no existe consenso en su definición, este es un punto importante al momento de explorar una intervención terapeútica. La mayoría de los ensayos clínicos que evalúan distintos tratamientos, no han demostrado cambiar ni mejorar el curso clínico de esta enfermedad; por ello, el soporte con 02 y la hidratación adecuada siguen siendo la piedra angular del cuidado respiratorio de lactantes con BA. Las HFNC representan una esperanza para los casos más graves. Es vital el rol de la prevención para evitar la diseminación intrahospitalaria y en la comunidad (Tabla 3).

Conflicto de interés: el autor es asesor médico de la empresa farmacéutica Glaxosmithkline en Chile.

\section{REFERÊNCIAS}

1. McLaurin KK, Farr AM, Wade SW, Diakun DR, Stewart DL. Respiratory syncytial virus hospitalization outcomes and costs of full-term and preterm infants. J Perinatol 2016;36:990-6. doi: 10.1038/jp.2016.113.

2. Fujiogi $M$, Goto $T$, Yasunaga $H$, Brown D, Mansbach J, Camargo C Jr. Trends in Bronchiolitis Hospitalizations in the United States: 2000-2016. Pediatrics 2019;144:e20192614. doi: 10.1542/peds.2012-3877.

3. Zorc JJ, Hall CB. Bronchiolitis: recent evidence on diagnosis and management. Pediatrics 2010;125:342-9. doi: 10.1542/peds.2009-2092.

4. Ralston S, Lieberthal A, Meissner HC, Alverson B, Baley J, Gadomski A, et al. Clinical practice guideline: the diagnosis, management, and prevention of bronchiolitis. Pediatrics 2014;134:e1474-502. doi: 10.1542/peds.2014-2742

5. Mansbach J, Espinola J, Macias C, Ruhlen M, Sullivan A, Camargo Jr C. Variability in the diagnostic labeling of nonbacterial lower respiratory tract infections: a multicenter study of children who presented to the emergency department. Pediatrics 2009;123:e573-e581. doi: 10.1542 peds.2008-1675

6. Nagakumar P, Doull I: Current therapy for bronchiolitis Arch Dis Child 2012:97:827-30. doi: 10.1136/archdischild-2011-301579

7. Hasegawa K, Jartti T, Mansbach JM. Respiratory syncytial virus genomic load and disease severity among children hospitalized with bronchiolitis: multicenter cohort studies in the United States and Finland. J Infect Dis 2015;211:1550-9. doi: 10.1093/infdis/jiu658

8. Brand HK, de Groot R, Galama JM, Monteleone K, Bonci E, Papoff $P$, et al. Infection with multiple viruses is not associated with increased disease severity in children with bronchiolitis. Pediatr Pulmonol 2012;47:393-400. doi: 10.1007/s00430-012-0233-6

9. Scagnolari C, Midulla F, Selvaggi C. Evaluation of viral load in infants hospitalized with bronchiolitis caused by respiratory syncytial virus. Med Microbiol Immunol 2012;201:311-7. doi: 10.1007/s00430-012-0233-6.

10. Mansbach JM, Piedra PA, Teach SJ. Prospective multicenter study of viral etiology and hospital length of stay in children with severe bronchiolitis. Arch Pediatr Adolesc Med 2012;166:700-6. doi: 10.1001/archpediatrics.2011.1669.

11. Jansen RR, Wieringa J, Koekkoek SM. Frequent detection of respiratory viruses without symptoms: toward defining clinically relevant cutoff values. J Clin Microbiol 2011:49:2631-6. doi: 10.1128/JCM.02094-10.

12. Hasegawa K, Linnemann RW, Avadhanula V, Mansbach J, Piedra P, Gern J, et al. Detection of respiratory syncytial virus and rhinovirus in healthy infants. BMC Res Notes 2015;8:718-24. doi: 10.1186/s13104-015-1695-6.

13. Ali S, Plint AC, Klassen TP. Bronchiolitis. In: Wilmott RW, Kendig EL, Boat TF, Bush A, Chernick V, editors. Kendig and Chernick's disorders of the respiratory tract in children. 8th edn. Elsevier Saunders; Philadelphia: 2012 pp. $443-52$.

14. Royal College of Paediatrics and Child Health national guidance. Scotland 2006, Scottish Intercollegiate Guidelines Network. A national clinical guidelines SICN 91. Bronchiolitis in Children. Available: https://www. nice.org.uk/guidance/ng9/documents/bronchiolitis-in-children-draft-guideline2. (acceso enero 2021).

15. Murray J, Bottle A, Sharland M, Sharland M, Modi N, Aylin $P$, et al. Medicines for Neonates Investigator G: Risk factors for hospital admission with RSV bronchiolitis in England: a population-based birth cohort study. PLoS One 2014;9:e89186. doi: 10.1371/journal.pone.0089186.

16. Schroeder AR, Destino LA, Ip W, Vukin E, Brooks R, Stoddard G, et al. Day of Illness and Outcomes in Bronchiolitis 
Hospitalizations. Pediatrics 2020;146(5):e20201537. doi: 10.1542/peds.2020-1537.

17. Rodríguez-Martínez CE, Castro-Rodriguez JA, Nino G, Midulla $F$. The impact of viral bronchiolitis phenotyping: Is it time to consider phenotype-specific responses to individualize pharmacological management?. Paediatr Respir Rev2020;34:53-8. doi: 10.1016/j.prrv.2019.04.003.

18. Schroeder AR, Marmor AK, Pantell RH, Newman TB. Impact of pulse oximetry and oxygen therapy on length of stay in bronchiolitis hospitalizations. Arch Pediatr Adolesc Med 2004:158:52730. doi: 10.1001/archpedi.158.6.527.

19. Choi J, Lee GL: Common pediatric respiratory emergencies Emerg Med Clin North Am 2012;30:529-63. do: 10.1016/j. emc.2011.10.009.

20. Parikh K, Hall M, Teach SJ: Bronchiolitis Management Before and After the AAP Guidelines. Pediatrics 2014;133:e1-7. doi: 10.1542/peds.2013-2005.

21. Ferronato AE, Gilio AE, Ferraro AA, Paulis M, Vieira SE: Etiological diagnosis reduces the use of antibiotics in infants with bronchiolitis. Clinics (Sao Paulo) 2012;67:1001-6. doi: 10.6061/clinics/2012(09)03.

22. Swingler $\mathrm{CH}$, Hussey $\mathrm{GD}$, Zwarenstein M. Randomised controlled trial of clinical outcome after chest radiograph in ambulatory acute lower-respiratory infection in children. Lancet 1998;351:404-8. doi: 10.1016/501406736(97)07013-X

23. Schuh S, Lalani A, Allen U, Manson D, Babyn P, Stephens $D$, et al. Evaluation of the utility of radiography in acute bronchiolitis. J Pediatr 2007;150:429-33. doi: 10.1016/j. jpeds.2007.01.005.

24. Mahabee-Gittens EM, Bachman DT, Shapiro ED, Dowd MD: Chest radiographs in the pediatric emergency department for children $<0 r=18$ months of age with wheezing. Clin Pediatr (Phila) 1999:38:395-9. doi: 10.1177/000992289903800703

25. Willson DF, Horn SD, Hendley JO, Smout R, Gassaway 」. Effect of practice variation on resource utilization in infants for viral lower respiratory illness. Pediatrics 2001;108:8515. doi: 10.1542/peds.108.4.851.

26. Wang EE, Law BJ, Boucher FD, Stephens D, Robinson L, Dobson S, et al. Pediatric Investigators Collaborative Network on Infections in Canada (PICNIC). Study of admission and management variation in patients hospitalized with respiratory syncytial viral lower respiratory tract infection. J Pediatr 1996;129:390-5. doi: 10.1016/s00223476(96)70071-9

27. Brand PLP, Vaessen-Verberne AAPH. Differences in management of bronchiolitis between hospitals in the Netherlands. Eur J Pediatr 2000;159:343-7. doi: 10.1007/ s004310051284.

28. Zielińska A, Jassem-Bobowicz JM, Kwiatkowska J. Oxygen therapy with high-flow nasal cannulas in children with acute bronchiolitis. Anaesthesiol Intensive Ther 2019;51:51-5. doi: 10.5603/AlT.2019.0010.

29. Rojas MX, Granados Rugeles C, Charry-Anzola LP. Oxygen therapy for lower respiratory tract infections in children between 3 months and 15 years of age. Cochrane Database Syst Rev 2009;21:CD005975. doi: 10.1002/14651858. CD005975.

30. Unger S, Cunningham S: Effect of oxygen supplementation on length of stay for infants hospitalized with acute viral bronchiolitis. Pediatrics 2008;121:470-5. doi: 10.1542/ peds.2007-1135.

31. Khoshoo V, Edell D. Previously healthy infants may have increased risk of aspiration during respiratory syncytial viral bronchiolitis. Pediatrics 1999:104:1389-90. doi: 10.1542/ peds.104.6.1389

32. Oakley E, Babl FE, Acworth J, Borland M, Kreiser D, Neutzeet $J$ et al. A prospective randomised trial comparing nasogastric with intravenous hydration in children with bronchiolitis (protocol): the comparative rehydration in bronchiolitis study (CRIB). BMC Pediatr 2010:10:37-40. doi:
10.1186/1471-2431-10-37.

33. Kennedy N, Flanagan N: Is nasogastric fluid therapy a safe alternative to the intravenous route in infants with bronchiolitis?. Arch Dis Child 2005;90:320-1. doi: 10.1136/ adc.2004.068916

34. Oakley E, Borland M, Neutze J, Aworth J, Krieser D, Dalziel $\mathrm{S}$, et al. Paediatric Research in Emergency Departments International Collaborative (PREDICT): Nasogastric hydration versus intravenous hydration for infants with bronchiolitis: a randomised trial. Lancet Respir Med 2013;:113-20. do: 10.1016/S2213-2600(12)70053-X.

35. Babl FE, Sheriff N, Neutze J, Borland M, Oakley E. Bronchiolitis management in pediatric emergency departments in Australia and New Zealand: a PREDICT study. Pediatr Emerg Care 2008;24:656-8. doi: 10.1097/PEC 0b013e318188498c

36. Gozal D, Colin AA, Jaffe M, Hochberg Z. Water, electrolyte, and endocrine homeostasis in infants with bronchiolitis. Pediatr Res 1990;27:204-9. doi: 10.1203/00006450199002000-00023.

37. Gadomski AM, Scribani MB. Bronchodilators for bronchiolitis. Cochrane Database Syst Rev. 2014;6 CD001266. doi 10.1002/14651858.CD001266 pub4

38. Hartling L, Bialy LM, Vandermeer B, Tjosvold L, Johnson D, Plint A, eta I. Epinephrine for bronchiolitis. Cochrane Database Syst Rev 2011;6:CD003123. doi: 10.1002/14651858 CD003123.pub3.

39. Fernandes RM, Bialy LM, Vandermeer B. Glucocorticoids for acute viral bronchiolitis in infants and young children Cochrane Database Syst Rev 2013:(6):CD004878. doi: 10.1002/14651858.CD004878.pub4.

40. van Woensel JB, van Aalderen WM, de Weerd W, Jansen N, van Gestel JP, Markhorst DG, et al. Dexamethasone for treatment of patients mechanically ventilated for lower respiratory tract infection caused by respiratory syncytial virus. Thorax 2003:58:383-7. doi: 10.1097/CCM. ob013e318218a030

41. Plint AC, Johnson DW, Patel H, Wiebe N, Correll R, Brant $\mathrm{R}$, et al. Pediatric Emergency Research Canada (PERC) Epinephrine and dexamethasone in children with bronchiolitis. N Engl J Med 2009:360:2079-89. doi: 10.1056 NEJMoa0900544

42. Plint AC, Grenon R, Klassen TP, Johnson DW. Bronchodilator and steroid use for the management of bronchiolitis in Canadian pediatric emergency departments. CJEM 2015;17:46-53. doi: 10.2310/8000.2013.131325.

43. Chao LC, Lin YZ, Wu WF, Huang FY. Efficacy of nebulized budesonide in hospitalized infants and children younger than 24 months with bronchiolitis. Acta Paediatr Taiwan 2003;44:332-5.

44. Luo Z, Liu E, Luo J. Nebulized hypertonic saline/salbutamol solution treatment in hospitalized children with mild to moderate bronchiolitis. Pediatr Int 2010;52:199-202. doi: 10.1111/j.1442-200X.2009.02941.x

45. Flores $\mathrm{P}$, Mendes AL, Neto AS. A randomized trial of nebulized $3 \%$ hypertonic saline with salbutamol in the treatmen of acute bronchiolitis in hospitalized infants. Pediatr Pulmonol 2016;51:418-25. doi: 10.1002/ppul.23306

46. Everard ML, Hind D, Ugonna K, Freeman J, Bradburn M, Cooper C, et al. SABRE: a multicentre randomised control trial of nebulised hypertonic saline in infants hospitalised with acute bronchiolitis. Thorax 2014;69:1105-12. do: 10.1136/thoraxinl-2014-205953.

47. Zhang L, Mendoza-Sassi RA, Klassen TP, Wainwright C Nebulized hypertonic saline for acute bronchiolitis: a systematic review. Pediatrics 2015;136:687-701. doi: 10.1542/ peds.2015-1914.

48. Maguire C, Cantrill H, Hind D, Bradburn M, Everard M. Hypertonic saline (HS) for acute bronchiolitis: systematic review and meta-analysis. BMC Pulm Med 2015;15:14854. doi: 10.1186/s12890-015-0140-x.

49. Brooks CG, Harrison WN, Ralston SL. Association between hypertonic saline and hospital length of stay in acute viral bronchiolitis: areanalysis of 2 meta-analyses. JAMA Pediatr 2016:170:577-84. doi: 10.1001/jamapediatrics.2016.0079.

50. Zhang L, Mendoza-Sassi RA, Klassen TP, Wainwright C. Nebulized Hypertonic Saline for Acute Bronchiolitis: A Systematic Review. Pediatrics 2015;136:687-701. doi: 10.1542/ peds.2015-1914.

51. Chandelia S, Kumar D, Chadha N, Jaiswal N. Magnesium sulphate for treating acute bronchiolitis in children up to two years of age. Cochrane Database Syst Rev 2020;12:CD012965. doi: 10.1002/14651858.CD012965. pub2.

52. King VJ, Viswanathan M, Bordley WC, Jackman A, Sutton $\mathrm{S}$, Lohr K, et al. Pharmacologic treatment of bronchiolitis in infants and children. Arch Pediatr Adolesc Med 2004;158:127-37. doi: 10.1001/archpedi.158.2.127.

53. Bradley IS, Conner JD, Compagiannis LS, Eiger LL. Exposure of health care workers to ribavirin during therapy for respiratory syncytial virus infections. Antimicrob Agents Chemother 1990;34:668-70. doi: 10.1128/aac.34.4.668.

54. Levine DA, Platt SL, Dayan PS, Macias C, Zorc J, Krief W, et al. Risk of serious bacterial infection in young febrile infants with respiratory syncytial virus infections. Pediatrics 2004;113:1728-34. doi: 10.1542/peds.113.6.1728.

55. Titus MO, Wright SW. Prevalence of serious bacterial infections in febrile infants with respiratory syncytial virus infections. Pediatrics 2003;112:282-4. doi: 10.1542/ peds.112.2.282.

56. Purcell K, Fergie J. Concurrent serious bacterial infections in 2396 infants and children hospitalized with respiratory syncytial virus lower respiratory tract infections. Arch Pediatr Adolesc Med 2002;156:322-4. doi: 10.1001/archpedi.156.4.322.

57. Davies HD, Matlow A, Petric M, Glazier R, Wang EE. Prospective comparative study of viral, bacterial and atypical organisms identified in pneumonia and bronchiolitis in hospitalized Canadian infants. Pediatr Infect Dis J 1996:15:371-5. doi: 10.1097/00006454-199604000-00017.

58. Shazberg G, Revel-Vilk S, Shoseyov D, Ben-Ami A, Klar A, Hurvitz H. The clinical course of bronchiolitis associated with otitis media. Arch Dis Child 2000;83:317-9. doi: 10.1136/adc.83.4.317.

59. McCallum GB, Morris PS, Chatfield MD, Maclennan C, White A, Sloots T. A single dose of azithromycin does not improve clinical outcomes of children hospitalised with bronchiolitis: a randomised, placebo-controlled trial. PLoS One 2013;8:e74316. doi: 10.1371/journal.pone.0074316.

60. Pinto LA, Pitrez PM, Luisi F. Azithromycin therapy in hospitalized infants with acute bronchiolitis is not associated with better clinical outcomes: a randomized, double-blinded, and placebo-controlled clinical trial. J Pediatr 2012:161:1104-8. doi: 10.1016/j.jpeds.2012.05.053.

61. Beigelman A, Isaacson-Schmid M, Sajol G. Baty J, Rodriguez $\mathrm{O}$, Leege $\mathrm{E}$, et al Randomized trial to evaluate azithromycin's effects on serum and upper airway IL-8 levels and recurrent wheezing in infants with respiratory syncytial virus bronchiolitis. J Allergy Clin Immunol 2015;135:1171-8. do: 10.1016/j.jaci.2014.10.001.

62. Roqué M, Giné-Garriga M, Granados C, Perrotta C, Vilaró J. Chest physiotherapy for acute bronchiolitis in paediatric patients between 0 and 24 months old. Cochrane Database Syst Rev. 2016 Feb 1;2(2):CD004873. doi: 10.1002/14651858.CD004873.pub5.

63. Gomes GR, Donadio MVF. Effects of the use of respiratory physiotherapy in children admitted with acute viral bronchiolitis. Arch Pediatr 2018;25:394-8. doi: 10.1016//. arcped.2018.06.004.

64. Jat KR, Mathew JL. Continuous positive airway pressure (CPAP) for acute bronchiolitis in children. Cochrane Database Syst Rev 2019;(1):CD010473. doi: 10.1002/14651858. CD010473.pub3.

65. Lazner MR, Basu AP, Klonin H. Non-invasive ventilation for 
severe bronchiolitis: Analysis and evidence. Pediatr Pulmonol 2012;47:909-16. doi: 10.1002/ppul.22513.

66. Zielińska A, Jassem-Bobowicz JM, Kwiatkowska J. Oxygen therapy with high-flow nasal cannulas in children with acute bronchiolitis. Anaesthesiol Intensive Ther 2019;51:51-5. doi: 10.5603/AlT.2019.0010

67. Lee JH, Rehder KJ, Williford L, Cheifetz IM, Turner DA: Use of high flow nasal cannula in critically ill infants, children, and adults: a critical review of the literature. Intensive Care Med 2012;39:247-57. doi: 10.1007/s00134-012-2743-5.

68. Klingenberg C, Pettersen M, Hansen EA, Gustavsen L, Dahl I, Leknessund $A$, et al. Patient comfort during treatment with heated humidified high flow nasal cannulae versus nasal continuous positive airway pressure: a randomised cross-over trial. Arch Dis Child Fetal Neonatal 2014;99:F134-7. doi: 10.1136/archdischild-2013-304525.

69. Beggs S, Wong ZH, Kaul S, Ogden K, Walters J, High-flow nasal cannula therapy for infants with bronchiolitis Cochrane Database Syst Rev 2014;1:CD009609. doi 10.1002/14651858.CD009609.pub2.

70. Hegde S, Prodhan P: Serious air leak syndrome complicating high-flow nasal cannula therapy: a report of 3 cases Pediatrics 2013;131:e939-e944. doi: 10.1542/peds.20113767.

71. Mansbach JM, Piedra PA, Stevenson MD, Sullivan A, Forgey $\mathrm{T}$, Clark $\mathrm{S}$, et al. Prospective multicenter study of children with bronchiolitis requiring mechanical ventilation. Pediatrics 2012;130:e492-e500. doi: 10.1542/peds.2012-0444.

72. Zamorano A, Mendez M. Bronquiolitis aguda: tratamiento de la insuficiencia respiratoria. Neumol Pediatr 2020;15:251-6

73. Mikalsen IB, Halvorsen T, Oymar K, The outcome after severe bronchiolitis is related to gender and virus. Pediatr Allergy Immunol 2012;23:391-8. doi: 10.1111/j.13993038.2012.01283.x.

74. Hyvarinen M, Plippo-Savolainen E, Korhonen K, Korppi M: Teenage asthma after severe infantile bronchiolitis or pneumonia. Acta Paediatr 2005;94:1378-83. doi: 10.1111/ j.1651-2227.2005.tb01807.x

75. Regnier SA, Huels J: Association between respiratory syncytial virus hospitalizations in infants and respiratory sequelae: systematic review and meta-analysis. Pediatr Infect Dis J 2013;32:820-6. doi: 10.1097/INF. Ob013e31829061e8.

76. Beigelman A, Bacharier LB: The role of early life viral bronchiolitis in the inception of asthma. Curr Opin Allergy Clin Immunol 2013;13:211-6. doi: 10.1097/ACI.0b013e32835eb6ef 